\title{
Effects of Aspergillus fumigatus culture filtrate on antifungal activity of human phagocytes in vitro
}

Takako Murayama, Ryoichi Amitani, Yumiko Ikegami, Rieko Kawanami, Woon Joo Lee, Ryuhei Nawada

\begin{abstract}
Background-Aspergillus fumigatus can colonise the airways and the lungs with localised underlying conditions and occasionally invade the surrounding lung tissues even in subjects without systemic predisposing factors, presumably by escaping the local host defences. The aim of this study was to investigate the effects of A fumigatus culture filtrate (ACF) on the activities of human phagocytesinhibition of germination of $A$ fumigatus spores by alveolar macrophages (AMs) and hyphal damage by polymorphonuclear leucocytes (PMNs)-which are the critical host defences against $A$ fumigatus.
\end{abstract}

Methods-Spores were incubated with $A M s$ at a ratio of $1: 1$ in a medium containing different concentrations of ACF for 10 hours at $37^{\circ} \mathrm{C}$. Spore germination was visualised with light microscopy and the inhibition rate was calculated. The percentage of hyphal damage caused by PMNs pretreated with various concentrations of ACF was measured by a colorimetric tetrazolium metabolic assay.

Results-The inhibition rate of spore germination by AMs cultured with medium alone (control) was $90(0.8) \%$ whereas that by AMs cultured with the medium containing $10 \%$ ACF was significantly $(p<0.05)$ reduced to $41.7(4.6) \%$. ACF suppressed the inhibition of spore germination in a dose dependent manner without altering the phagocytosing activity against the spores. The percentage of hyphal damage caused by PMNs pretreated with medium-199 (control) was 78.1 (2.3)\% compared with $65.3(2.8) \%$ when PMNs were pretreated with $50 \%$ ACF $(p<0.05)$. Conclusions-A fumigatus releases biologically active substance(s) which suppress the inhibition of spore germination by AMs and also suppress PMN mediated hyphal damage, and thus may contribute to the pathogenicity of this fungus.

(Thorax 1998;53:975-978)

Keywords: Aspergillus fumigatus; human phagocytes; host defence

Aspergillus species, most commonly Aspergillus fumigatus, are responsible for a wide range of diseases including saprophytic colonisation, chronic necrotising aspergillosis, and rapidly invasive diseases. ${ }^{12}$ Although acute progressive and invasive diseases occur most commonly in systemic immunocompromised hosts, A fumi- gatus frequently colonises damaged lungs and airways including healed tuberculosis, bronchiectasis and cystic fibrosis, and occasionally invades the surrounding lung tissues even in patients without apparently compromised systemic host defences. ${ }^{12}$ However, the pathogenic mechanisms and substances are still poorly understood.

It has been proposed that host defences against $A$ fumigatus consist of mucociliary clearance in the airways, alveolar macrophages (AMs), and polymorphonuclear leucocytes (PMNs). AMs can ingest the inhaled spores of A fumigatus, inhibit them from germination, and kill them. The germinated spores and hyphae that escape the mucociliary clearance and AMs can be killed by PMNs, mainly by an oxidative mechanism. ${ }^{3}$ These lines of defence cooperate in elimination of the fungus.

We have previously reported that $A$ fumigatus culture filtrate (ACF) causes damage to respiratory ciliated epithelium by reducing ciliary beat frequency, and suppresses human phagocyte function including PMN migration towards formyl-methionyl-leucyl-phenylalanine and $\mathrm{O}_{2}$ release from PMNs in response to phorbol myristate acetate, and concluded that $A$ fumigatus produces several substances which suppress phagocyte function and mucociliary clearance..$^{5-7}$

In the present study we have investigated the effects of ACF on inhibition of spore germination by human AMs and hyphal damage by human PMNs, in addition to the effects of $\mathrm{ACF}$ on ingestion of spores by AMs and the release of $\mathrm{O}_{2}^{-}$from PMNs in response to $A$ fumigatus hyphae.

\section{Methods}

FUNGUS

A fumigatus, YN strain, clinically isolated from the sputum of a patient with invasive pulmonary aspergillosis, was used in the present study. The strain was stored on potato dextrose agar (PDA) (Difco Laboratories, Detroit, Michigan, USA) slants at $-80^{\circ} \mathrm{C}$. Spores were obtained by scraping the surface of the slants, suspended in phosphate buffered saline (PBS), and kept at $4^{\circ} \mathrm{C}$. To induce spore germination a suspension of $1.0 \times 10^{6}$ spores in RPMI-1640 (Gibco BRL, Paisley, UK) was incubated in polypropylene tubes (Falcon, Becton Dickinson, Lincoln, New Jersey, USA) for 16 hours at $30^{\circ} \mathrm{C}$ and for an additional two hours at $37^{\circ} \mathrm{C} .{ }^{8}$ After incubation for 18 hours almost all spores developed into hyphae with a length of 10-30 $\mu \mathrm{m}$, but not branched. The hyphae were washed with PBS, vigorously vortexed, and used in the PMN experiments. 
PREPARATION OF A FUMIGATUS CULTURE FILTRATE

A fumigatus culture filtrate (ACF) was prepared as described previously. ${ }^{5}$ Spores $\left(2 \times 10^{7}\right)$ of YN strain were cultured in flasks containing $300 \mathrm{ml}$ of medium-199 (Gibco BRL) at $37^{\circ} \mathrm{C}$. After five days of culture the fungi were removed by passing the medium through gauze, and ACF was sterilised by passage through Millex-GV $0.22 \mu \mathrm{m}$ millipore filters (Millipore Products, Bedford, Massachusetts, USA) and stored at $-80^{\circ} \mathrm{C}$.

PHAGOCYTES

Human AMs were isolated from bronchoalveolar lavage (BAL) fluid of six healthy adult volunteers. BAL was performed with $4 \times 50 \mathrm{ml}$ sequential volumes of saline at $37^{\circ} \mathrm{C}$. The recovery rate of BAL fluid was 60-65\%, and Giemsa staining of cytospin preparations of BAL suspensions showed that more than $90 \%$ comprised AMs.

PMNs were prepared from the heparinised venous blood obtained from eight healthy adult volunteers by dextran sedimentation followed by centrifugation over Ficoll gradient and hypotonic lysis of erythrocytes. After treatment with ACF, the viability of cells was more than 95\% as assessed by the trypan blue exclusion test. Cell damage was checked by release of lactate dehydrogenase (LDH) and no difference was found in LDH concentration whether or not they had been treated with ACF.

INGESTION OF SPORES BY AM

Ingestion of spores was assessed as described previously. ${ }^{7}$ Spores of YN strain were added to the AM suspensions $\left(2 \times 10^{6} / \mathrm{ml}\right)$ in medium199 containing ACF $(0 \%, 5 \%$, and $10 \%)$ at an AM:spore ratio of 1:5. Each mixture was incubated in a water bath with shaking at $37^{\circ} \mathrm{C}$ for 120 minutes. Immediately after incubation, all tubes were washed with cold $0.1 \%$ EDTAPBS. Cells were attached to a glass slide by the cytospin procedure and stained with MayGrunwald-Giemsa. Both percentage phagocytosis and phagocytic index were calculated by counting 500 AMs per glass slide under a light microscope. Percentage phagocytosis was defined as the percentage of AMs with one or more spores ingested or attached. The phagocytic index was the mean number of spores that had been ingested by or attached to100 AMs.

INHIBITION OF SPORE GERMINATION BY AM

The ability of AMs to inhibit spore germination was determined by a modification of the procedure of Waldorf et al. ${ }^{9}$ One millilitre aliquots of cell suspension in culture medium (RPMI-1640 with 5\% heat inactivated AB serum) were seeded into multiwell tissue culture plates (Falcon) and incubated at $37^{\circ} \mathrm{C}$

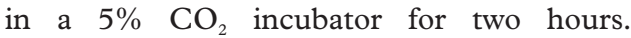
Non-adherent cells were discarded by washing with RPMI-1640 and the culture medium containing various concentrations of $\mathrm{ACF}$ $(0 \%, 1 \%, 3 \%, 5 \%$, and $10 \%)$ was added to each well to form monolayers of AMs at a concentration of $5 \times 10^{5} /$ well. AMs were challenged with spores in a ratio of $1: 1$ and centri- fuged for five minutes at $100 \mathrm{~g}$ to facilitate contact between the spores and AMs. Incubation was continued for a total of 10 hours at $37^{\circ} \mathrm{C}$ in the $\mathrm{CO}_{2}$ incubator, after which the plates were centrifuged, the supernatant discarded, and $0.5 \mathrm{ml}$ of $2.5 \%$ deoxycholate (Sigma, St Louis, Missouri, USA) added to each well to lyse AMs. The plates were stained with lactophenol cotton blue and an inverted microscope (Olympus Optical Co, Tokyo, Japan) was used to visualise spore germination. The inhibition rate was calculated from the percentage of spores which did not germinate out of a total of 500 examined per well.

RELEASE OF $\mathrm{O}_{2}{ }^{-}$FROM PMNS IN RESPONSE TO HYPHAE

Release of $\mathrm{O}_{2}^{-}$from $\mathrm{PMN}$ in response to $A$ fumigatus hyphae was assessed by superoxide dismutase (SOD) inhibitable reduction of cytochrome C. ${ }^{10}$ Briefly, PMNs were preincubated with medium-199 containing various concentrations of $\mathrm{ACF}(0 \%, 10 \%, 30 \%$, and $50 \%$ ) for one hour at $37^{\circ} \mathrm{C}$ and then washed with PBS. PMNs were incubated with hyphae at a ratio of $1: 1$ in $1 \mathrm{ml}$ of Hank's balanced salt solution (HBSS) containing cytochrome C $(120 \mu \mathrm{mol})$ (Sigma), with or without $50 \mu \mathrm{g}$ $\operatorname{SOD}$ (Sigma), in a shaking water bath at $37^{\circ} \mathrm{C}$ for one hour. These reaction tubes were centrifuged and $\mathrm{O}_{2}^{-}$production was assessed in the supernatants as the difference in absorption from the control at $550 \mathrm{~nm}$ measured on a spectrophotometer (Hitachi U-3210, Hitachi Ltd., Tokyo, Japan), and amount of $\mathrm{O}_{2}^{-}$in nanomoles released per $10^{6} \mathrm{PMNs}$ was calculated. All experiments were performed in quadruplicate.

PMN-INDUCED HYPHAL DAMAGE

Damage of the hyphae caused by PMNs was assessed by a colorimetric assay. ${ }^{8}$ PMNs were pretreated with various concentrations of ACF $(0 \%, 10 \%, 30 \%$, and $50 \%)$ for one hour and washed with PBS. PMNs $\left(6 \times 10^{6}\right)$ were resuspended in HBSS and added to hyphae in a final volume of $1 \mathrm{ml}$ containing $5 \%$ heat inactivated $\mathrm{AB}$ serum at a PMN:hyphae ratio of $4: 1$. After one hour of incubation at $37^{\circ} \mathrm{C}$ in a $\mathrm{CO}_{2}$ incubator the PMNs were lysed with distilled water. The hyphae were then washed with distilled water and resuspended in $0.4 \mathrm{ml}$ of $0.5 \mathrm{mg} / \mathrm{ml}$ 2,3-bis-(2-methoxy-4-nitro-5-sulphenyl)-(2H)tetrazolium-5-carboxanilide (XTT; Sigma) supplemented with $40 \mu \mathrm{g} / \mathrm{ml}$ coenzyme Q (Sigma). Assay tubes were further incubated for one hour at $37^{\circ} \mathrm{C}$ in a water bath and $100 \mu \mathrm{l}$ from each supernatant was transferred to the wells of microtitre plates. Absorption at $450 \mathrm{~nm}$ was determined using an ELISA plate reader (Flow Laboratories). The percentage of hyphal damage was defined as $\left[1-\left(\mathrm{A}_{450}\right.\right.$ of fungi incubated with $\mathrm{PMN}-\mathrm{A}_{450}$ of $\mathrm{PMN}$ alone)/A $\mathrm{A}_{450}$ of fungi incubated without $\mathrm{PMN}] \times 100 \%$.

ANALYSIS OF DATA

All data are presented as mean (SE) values. AM experiments were carried out in duplicate and PMN experiments in quadruplicate on four or five separate occasions. The raw data 


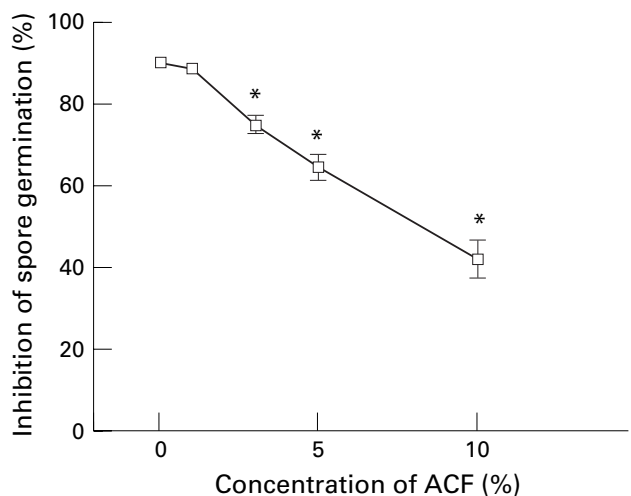

Figure 1 Effect of A fumigatus culture filtrate $(A C F)$ on inhibition of spore germination by human alveolar macrophages (AMs). AMs co-cultured with different concentrations of $A C F$ were challenged with spores at $37^{\circ} \mathrm{C}$ for 10 hours. The AM:spore ratio was 1:1. The percentage of inhibition was determined by calculating the number of spores which did not germinate. The results represent the mean (SE) of four separate experiments, each performed in duplicate. ${ }^{\star} p<0.05$ compared with control (medium alone).

were subjected to repeated measures analysis of variance (ANOVA) and the Dunnett's pairwise multiple comparison $t$ test. A p value of less than 0.05 was considered to be significant between control and ACF treatment.

\section{Results}

EFFECT OF ACF ON INHIBITION OF SPORE GERMINATION BY AM

AM cultured with medium alone (control) inhibited germination of $A$ fumigatus spores effectively with an inhibition rate of $90(0.8) \%$. On the other hand, treatment with $5 \%$ and $10 \%$ ACF led to reductions of $29 \%$ and $54 \%$, respectively, in the inhibition rate compared with the control ( $p<0.05$; fig 1$)$. The number of AMs recovered from culture supernatant in each well after 10 hours of incubation was always less than $4 \times 10^{4}$ which indicated that the suppressive effect of ACF was not a consequence of loss of AMs. To determine whether ACF has a direct effect on spore germination, spores were incubated in AM-free medium containing ACF at concentrations similar to those used in the AM experiments. ACF did not show any direct effects on spore germination, and germination rates incubated with or without ACF were always $>99 \%$.

The effect of ACF on the phagocytic activity of AMs against spores was also evaluated. Percentage phagocytosis and the phagocytic index were $69.1(7.0) \%$ and 155.6 (45.8) in medium alone, 73.6 (5.0)\% and 179.5 (54.1) when pretreated with $5 \% \mathrm{ACF}$, and $72.0(4.4) \%$ and 172.8 (52.1) when pretreated with $10 \%$ ACF. Thus, 5-10\% ACF had no significant effect on either AM mediated percentage phagocytosis or percentage phagocytic index $(p>0.05)$. It is therefore very likely that the suppressive effect of ACF on the inhibition of spore germination described above was not associated with its effect on the ingestion of spores.

EFFECT OF ACF ON $\mathrm{O}_{2}^{-}$RELEASE FROM PMN STIMULATED WITH HYPHAE

The effect of ACF on the oxidative metabolic burst of PMN, measured as the release of $\mathrm{O}_{2}^{-}$in response to non-opsonised $A$ fumigatus hyphae,

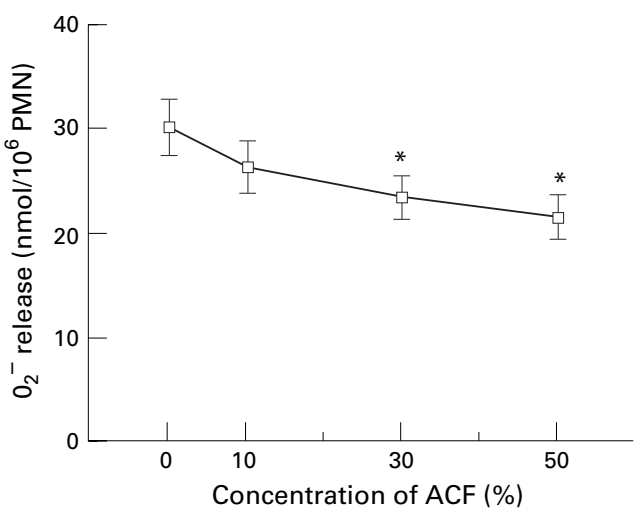

Figure 2 Effect of A fumigatus culture filtrate (ACF) on $\mathrm{O}_{2}^{-}$release by human polymorphonuclear leucocytes (PMNs) in response to A fumigatus hyphae. PMNs were pretreated with various concentrations of $A C F$ at $37^{\circ} \mathrm{C}$ for one hour. $\mathrm{O}_{2}^{-}$release was assessed by cytochrome $C$ reduction assay at a PMN:hyphae ratio of 1:1 and expressed as nmol/106 PMNs/h. The results represent the mean (SE) of five separate experiments, each performed in quadruplicate. ${ }^{*} p<0.05$ compared with control (medium-199).

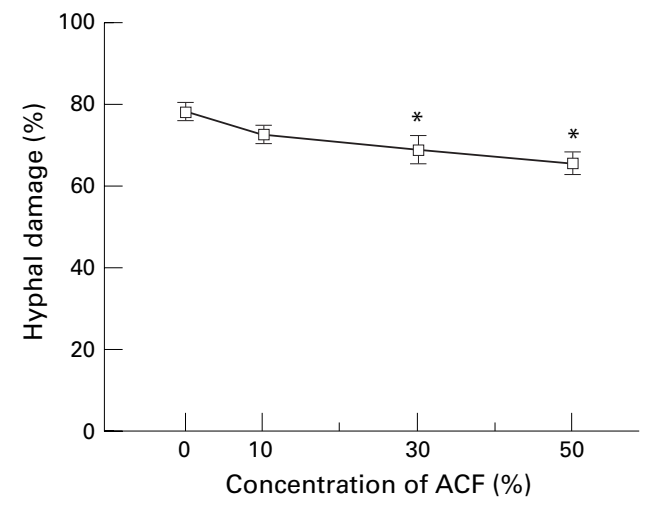

Figure 3 Effects of A fumigatus culture filtrate (ACF) on hyphal damage by human PMNs. PMN suspensions pretreated with various concentrations of $A C F$ were co-cultured with $A$ fumigatus hyphae at $37^{\circ} \mathrm{C}$ for one hour. The PMN:hyphae ratio used in this experiment was 4:1.

The percentage of hyphal damage was examined by a colorimetric assay using $(2,3)$-bis- (2-methoxy-4nitro-5-sulphenyl)-(2H)-tetrazolium-5-carboxanilide (XTT). The results represent the mean (SE) of five separate experiments, each performed in quadruplicate. ${ }^{\star} p<0.05$ compared with control (medium-199).

was examined. As shown in fig 2, pretreatment with $30 \%$ and $50 \%$ ACF for one hour significantly suppressed $\mathrm{O}_{2}^{-}$release in response to hyphae by $22 \%$ and $29 \%$, respectively $(\mathrm{p}<0.05)$. Since spontaneous $\mathrm{O}_{2}^{-}$release was not affected by ACF, and ACF itself did not stimulate $\mathrm{O}_{2}^{-}$release of PMNs, ${ }^{7}$ this suppressive effect of ACF was not due to exhaustion of PMNs by ACF.

EFFECT OF ACF ON PMN INDUCED HYPHAL

DAMAGE

We assessed the hyphal damage using the XTT colorimetric metabolic assay, a rapid and quantitative method for assessment of fungal cell damage. ${ }^{8}$ The effects of ACF on PMN induced damage of $A$ fumigatus hyphae are shown in fig 3. Pretreatment with $30 \%$ and $50 \%$ ACF for one hour significantly suppressed the percentage of hyphal damage by $12 \%$ and $16 \%$, respectively $(\mathrm{p}<0.05)$. When PMNs and hyphae were incubated with ACF throughout 
the one hour experimental period without pretreatment, ACF showed the same suppressive effect on the PMN induced hyphal damage (data not shown). When hyphae were incubated in PMN-free medium containing ACF at the same concentrations as used in the PMN experiments, ACF did not affect the metabolic activity of the hyphae as determined by XTT assays (data not shown).

\section{Discussion}

The results of this study show that ACF suppresses inhibition of spore germination of A fumigatus by human AMs without altering their phagocytosing activity against the spores, and that ACF also suppresses human PMN induced $A$ fumigatus hyphal damage apparently parallel with $\mathrm{O}_{2}{ }^{-}$release in response to hyphae. Although the present study was performed using a single strain of $A$ fumigatus, limited experiments carried out with other clinical isolates of $A$ fumigatus have shown similar results (data not shown).

Roilides et al reported that PMNs can respond to and damage Aspergillus hyphae irrespective of opsonisation, and that serum independent receptors of PMNs may contribute to attachment to Aspergillus hyphae and stimulation of the oxidative burst in the alveoli where serum opsonisation is poor. ${ }^{10}$ Warren et al found that PMNs can damage hyphae by releasing reactive oxygen intermediates and that the degree of the damage correlates with the duration and the levels of oxidant production. ${ }^{1}$ In this study we found that $\mathrm{ACF}$ suppresses $\mathrm{O}_{2}^{-}$release from PMNs in response to non-opsonised hyphae and also suppresses PMN mediated hyphal damage at similar concentrations. We assume that $A$ fumigatus derived products circumvent the host defence network, thus enhancing the chance of colonisation, proliferation, and invasion of $A$ fumigatus in human tissues.

It has been suggested that secondary metabolites, especially gliotoxin and extracellular proteases, may contribute to the pathogenic potential of $A$ fumigatus. ${ }^{6}{ }^{11-13}$ Müllbacher et al reported that a three day culture of $A$ fumigatus generated metabolites with antiphagocytic activity (inhibition of phagocytosis by mouse peritoneal exudate cells, inhibition of adherence to plastic surfaces by rodent peritoneal macrophages and AMs, reduction of the basal rate of $\mathrm{H}_{2} \mathrm{O}_{2}$ production by human PMNs), and they identified one of the biologically active components as gliotoxin. ${ }^{11}{ }^{12}$ Slight et al reported that $A$ fumigatus spore diffusates obtained from three hour culture supernatants reduced phagocytic activity (inhibition of oxidative burst by rat AMs and inhibition of production of tumour necrosis factor $\alpha$ by rat AMs). They suggested that the active component of spore diffusate was of small molecular weight $(<10 \mathrm{kD})$ and might have a carbohydrate component. ${ }^{14}{ }^{15}$ However, to our knowledge there have been very few studies to date that have reported the suppressive effects of Aspergillus products on the antifungal activities of human phagocytes. This study may be the first to demonstrate the suppressive effects of Aspergillus products on antifungal host defences by both human AMs and PMNs.

Amitani et al isolated the factors responsible for causing damage to the human respiratory ciliated epithelium from ACF and identified one of the most potent substances as gliotoxin. ${ }^{6}$ We have previously suggested that gliotoxin should be a candidate for the antiphagocytic substances in $\mathrm{ACF}^{7}$ Richard et al detected gliotoxin in the lung tissue of turkey poults infected with $A$ fumigatus and suggested the involvement of gliotoxin in the pathogenesis of aspergillosis. ${ }^{16}$ These findings suggest that gliotoxin should be one of the most important substances contributing to fungal growth in human tissues.

Additional studies are in progress to clarify which of various $A$ fumigatus derived substances including gliotoxin are the most important in suppressing human phagocytic function.

We thank Professor R D Diamond, Section of Infectious Diseases, University Hospital, Boston University for his helpful advice regarding the assay of hyphal damage and Dr S Ishimura, Tsurumi University for his critical advice regarding the statistical analysis.

1 Warren BG. The spectrum of pulmonary aspergillosis. $\mathcal{f}$ Thorac Imaging 1992; 7:56-74

2 Binder RE, Faling LJ, Pugatch RD, et al. Chronic necrotizing pulmonary aspergillosis: discrete clinical entity. Medicine 1982;61:109-24.

3 Calderone R, Diamond RD, Senet J-M, et al. Host cell-fungal interactions. F Med Vet Mycol 1994;32:151-68. 4 Schaffner A. Macrophage-Aspergillus interactions. Immunol Ser 1994;60:545-52.

5 Amitani R, Murayama T, Nawada R, et al. Aspergillus culture filtrates and sputum sols from pulmonary aspergillosis cause damage to human respiratory ciliated epithelium in vitro. Eur Respir f 1995;8:1681-7.

6 Amitani R, Taylor G, Elezis E-N, et al. Purification and characterization of factors produced by Aspergillus fumigatus which affect human ciliated respiratory epithelium. Infect Immun 1995;63:3266-71.

7 Murayama T, Amitani R, Ikegami Y, et al. Suppressive effects of Aspergillus fumigatus culture filtrates on human alveolar macrophages and polymorphonuclear leukocytes. Eur Respir f 1996;9:293-300.

8 Meshulam T, Levitz SM, Christin L, et al. A simplified new assay for assessment of fungal cell damage with the tetrazolium dye, $(2,3)$-bis-(2-methoxy-4-nitro-5-sulphenyl)-(2H)tetrazolium-5-carboxanilide (XTT). F Infect Dis 1995;172: tetrazoliim $1153-6$.

9 Waldorf AR, Levitz SM, Diamond RD. In vivo bronchoalveolar macrophage defense against Rhizopus oryzae and Aspergillus fumigatus. F Infect Dis 1984;150:752-60.

10 Roilides E, Mertins S, Eddy J, et al. Enhancement of oxidative response and damage caused by human neutrophils to Aspergillus fumigatus hyphae by granulocyte colonystimulating factor and gamma interferon. Infect Immun 1993;61:1185-93.

11 Eichner RD, Salami MAL, Wood PR, et al. The effect of gliotoxin upon macrophage function. Int f Immunopharmacol 1986;8:789-97.

12 Müllbacher A, Waring P, Eichner RD. Identification of an agent in cultures of Aspergillus fumigatus displaying anent in cultures of Aspergilus fumigatus displaying anti-phagocytic and immunom

13 Robinson BWS, Venaille TJV, Mendis AHW, et al. Allergens as proteases: an Aspergillus fumigatus proteinase directly induces human epithelial cell detachment. $\mathcal{f}$ Allergy Clin Immunol 1990;85:726-31.

14 Nicholson WJ, Slight J, Donaldson K. Inhibition of the transcription factor NF- $\mathrm{KB}$ and AP-1 underlies loss of cytokine gene expression in rat alveolar macrophages treated with a diffusible product from the spores of Aspergillus fumigatus. Am F Respir Cell Mol Biol 1996;15:8896.

15 Slight J, Nicholson WJ, Mitchell CG, et al. Inhibition of the alveolar macrophage oxidative burst by diffusible component from the surface of the spores of the fungus Aspergillus fumigatus. Thorax 1996;51:389-96.

16 Richard JL, DeBey MC. Production of gliotoxin during the pathogenic states in turkey poults by Aspergillus fumigatus pathogenic states in turkey poults by Aspergith 